\title{
ON LIE ALGEBRAS OF TYPE $E_{6}$
}

\author{
BY J. C. FERRAR ${ }^{1}$
}

Communicated by G. D. Mostow, September 21, 1966.

Introduction. In this note, we investigate, omitting details, the structure of Lie algebras of type $E_{6}$ over arbitrary fields of characteristic other than two or three, introducing certain invariants for such algebras and studying the implications these invariants have for the structure of the algebras in question. As a consequence of this investigation, we obtain, producing constructively a representative of each isomorphism class, a complete classification of algebras of type $E_{6}$ over finite, real closed, or $p$-adic fields, as well as partial results for algebraic number fields. Since every Lie algebra of type $E_{6}$ over $\Phi$ has a finite, Galois, splitting field $P \supseteq \Phi,[1]$, we restrict our attention, without loss of generality, to a particular pair of fields $P$ and $\Phi$, $P$ finite, Galois over $\Phi$ with group $G$, and to the collection of Lie algebras of type $E_{6}$ over $\Phi$ which are split by $P$.

Realization of the split $E_{6}$. Let $g_{0}$ be a split exceptional central simple Jordan algebra over $\Phi, \mathfrak{J}=\mathfrak{J}_{0} \otimes_{\Phi} P$, and $V$ the $P$-space of all

$$
x=\left(\begin{array}{ll}
\alpha_{1} & a_{1} \\
a_{2} & \alpha_{2}
\end{array}\right), \alpha_{i} \in P, a_{i} \in \mathfrak{J} .
$$

$V$, with quartic form

$$
\begin{aligned}
q(x)=8\left(a_{1} \times a_{1}, a_{2} \times a_{2}\right)-8 \alpha_{1} N\left(a_{1}\right)-8 \alpha_{2} N\left(a_{2}\right) & \\
& -2\left(\left(a_{1}, a_{2}\right)-\alpha_{1} \alpha_{2}\right)^{2},
\end{aligned}
$$

$(a, b)$ the trace bilinear form of $\mathcal{g}, N(a)$ the generic norm on $\mathcal{g}, \times$ the product defined in [4], is a module for the split Lie algebra of type $E_{7}$ [4], [8]. The algebra $\&\left(V, V_{0}\right)=\left\{L \in \operatorname{Hom}(V, V) \mid V_{0} L=0, L\right.$ skew with respect to the linearized $q(x)\}, V_{0}$ the subspace of $V$ of diagonal elements, is a split Lie algebra of type $E_{6}$. The semiautomorphisms of $\mathfrak{L}\left(V, V_{0}\right)$ are described by

THEOREM 1. $A(s)$ is an s-semiautomorphism of $\mathcal{L}\left(V, V_{0}\right)$ if and only if there is a permutation $\pi$ of $\{1,2\}$, an element $\gamma \in P^{*}$, and an $s$ semilinear transformation $T(s)$ on d satisfying $N(x T(s))=\mu N(x)^{s}$ for all $x \in \mathcal{J}$, such that

1 The contents of this paper form part of the author's dissertation at Yale University, written under the direction of Professor N. Jacobson while the author was a National Science Foundation Graduate Fellow. 


$$
L A(s)=[\pi, \gamma, T(s)]^{-1} L[\pi, \gamma, T(s)] \stackrel{\text { def }}{=} L[\pi, \gamma, T(s)] \nu
$$

for all $L \in \mathcal{L}\left(V, V_{0}\right),[\pi, \gamma, T(s)]$ the s-semilinear transformation on $V$ such that

$$
\left(\begin{array}{ll}
\alpha_{1} & a_{1} \\
a_{2} & \alpha_{2}
\end{array}\right)[\pi, \gamma, T(s)]=\left(\begin{array}{ll}
\alpha_{1 \pi}^{*} \mu^{-1} \gamma^{2} & a_{1 \pi} T(s) \\
a_{2 \pi} T(s)^{*-1} \gamma & \alpha_{2 \pi}^{s} \mu \gamma^{-1}
\end{array}\right)
$$

where $*$ is the transpose with respect to $(a, b)$ in $\mathrm{g}$.

Invariants. If $\mathcal{L}$ is of type $E_{\mathfrak{B}}$ over $\Phi, \mathfrak{L}_{P}=\mathfrak{L} \otimes_{\Phi} P$ split, we may, up to isomorphism, assume that $\mathfrak{L}$ is a $\Phi$-form of $\mathscr{L}\left(V, V_{0}\right)$ and hence, by standard results, there is a homomorphism $s \rightarrow A(s)$ of $G$ into the group of $s$-semiautomorphisms of $\&\left(V, V_{0}\right)$ such that

$$
\mathfrak{L}=\left\{L \in \mathscr{L}\left(V, V_{0}\right) \mid L A(s)=L \text { for all } s \in G\right\} .
$$

By Theorem 1, $A(s)=[\pi(s), \gamma(s), T(s)]^{\nu}$ for each $s$ and one sees easily that $s \rightarrow \pi(s)$ is a homomorphism of $G$ into $S_{2}$ which is invariant under isomorphism of $\&$. We call $\&$ of type $E_{6 \mathrm{II}}$ if $\pi(G)=1$, of type $E_{6 \mathrm{II}}$ if $\pi(G)=S_{2}$. If $\&$ is of type $E_{6 \mathrm{II}}$, there is a unique quadratic extension $\Delta$ of $\Phi$ (the canonical $E_{6 \mathrm{I}}$ extension of $\Phi$ for $\mathcal{L}$ ) such that $\mathcal{L}_{\Delta}$ is of type $E_{\text {6I. }}$.

Considering $\mathscr{L}$ as $\Phi$-subalgebra of $\&\left(V, V_{0}\right) \subseteq \operatorname{Hom}(V, V)$, we define $\mathscr{L}^{*}$ to be the enveloping associative algebra of $\mathscr{L}$ in $\operatorname{Hom}(V, V)$ and observe that $\mathfrak{L}^{*}$ is an invariant of the isomorphism class of $\mathscr{L}$.

Finally, defining $\mathfrak{T}^{\prime}\left(V, V_{0}\right)=\left\{[1, \gamma, T] \mid T\right.$ linear, $\left.\gamma \in P^{*}\right\}$ and $K=\left\{[1, \gamma, \alpha I] \mid I\right.$ the identity on $\left.\mathcal{g}, \alpha \in P^{*}\right\}$ we have an exact sequence

$$
1 \rightarrow K \rightarrow \mathfrak{N t}^{\prime}\left(V, V_{0}\right) \stackrel{\nu}{\rightarrow} \operatorname{Aut}^{\prime} \mathfrak{\&}\left(V, V_{0}\right) \rightarrow 1
$$

where Aut' $\&\left(V, V_{0}\right)$ is the image of $\mathfrak{T}^{\prime}\left(V, V_{0}\right)$ under $\nu$. This can be made into a sequence of $G$-groups by defining the action of $s \in G$ on $\mathfrak{T I}^{\prime}\left(V, V_{0}\right)$ (resp. Aut' $\left.\&\left(V, V_{0}\right)\right)$ to be conjugation by $[\pi(s), 1, I(s)]$ (resp. $\left.[\pi(s), 1, I(s)]^{\nu}\right), I(s)$ the $s$-semilinear extension of the identity on $g_{0}$ to $\mathcal{g}, s \rightarrow \pi(s)$ the homomorphism associated with $\mathcal{L}$. Since $K$ is contained in the center of $\mathfrak{T}^{\prime}\left(V, V_{0}\right)$ and one can show $H_{\pi}^{1}(G, K)=1$, we have the exact cohomology sequence

(2) $1 \rightarrow H_{\pi}^{1}\left(G, \operatorname{gic}^{\prime}\left(V, V_{0}\right)\right) \rightarrow H_{\pi}^{1}\left(G, \mathrm{Aut}^{\prime} \mathfrak{\&}\left(V, V_{0}\right)\right) \rightarrow H_{\pi}^{2}(G, K)$.

Identifying, in the usual manner [6], the elements of $H_{\pi}^{1}(G$, $\operatorname{Aut}^{\prime} \mathfrak{L}\left(V, V_{0}\right)$ ) with the equivalence classes of isomorphic forms of $\mathfrak{L}\left(V, V_{0}\right)$ having associated homomorphism $\pi: G \rightarrow S_{2}$, we define $\Gamma(\mathcal{L}) \in H_{\pi}^{2}(G, K)$ to be the image of the element of $H_{\pi}^{1}\left(G, \operatorname{Aut}^{\prime} \&\left(V, V_{0}\right)\right)$ 
corresponding to the class containing $\mathscr{L}$, in $(2) . \Gamma(\mathscr{L})$ is not, in general, an invariant of the complete isomorphism class containing $\mathscr{L}$.

We have the following results describing and relating the invariants.

TheOREM 2. (a) $\mathscr{L}$ is of type $E_{6 \mathrm{I}}$ over $\Phi$ if and only if $\mathfrak{L}^{*} \cong \mathfrak{V} \oplus \mathfrak{B}^{\prime}$, $\mathfrak{B}^{\prime}$ antiisomorphic to $\mathfrak{B}, \mathfrak{B}$ central simple of exponent three, degree 27 over $\Phi$.

(b) $\mathcal{L}$ is of type $E_{6 \mathrm{II}}$ with canonical $E_{6 \mathrm{I}}$ extension $\Delta$ of $\Phi$ if and only if $\mathfrak{L}^{*} \cong \mathfrak{B}, \mathfrak{B}$ central simple associative of exponent three, degree 27 over $\Delta$ with involution of the second kind over $\Phi$.

THEOREM 3. $\Gamma(\mathfrak{L})=1$ if and only if $\mathfrak{L}^{*}$ is isomorphic to either $\Phi_{27} \oplus \Phi_{27}$ or to $\Delta_{27}$, depending on the $E_{6}$ type of $\mathcal{L}$.

Since $\mathfrak{L}^{*}$ is invariant under isomorphism, so is the property $\Gamma(\mathcal{L})=1$.

Construction of algebras. Let $g$ be an arbitrary exceptional central simple Jordan algebra over $\Phi, N(x)$ the generic norm form of $\mathcal{g}$, $N(x, y, z)$ the linearized norm form. The set of all $L \in \operatorname{Hom}(\mathcal{J}, \mathcal{J})$ such that $N(x L, x, x)=0$ for all $x \in \mathcal{J}$ is an algebra $\mathscr{L}(\mathcal{J})$ of type $E_{6}$ [3] and can be written as the direct sum $T(\mathcal{g})+\theta(\mathcal{g}), T(\mathcal{g})$ the set of right multiplications by elements of trace zero in $\mathcal{g}, \theta(\mathcal{J})$ the derivation algebra of $g$. Since $[T(\mathcal{J}), T(\mathcal{J})] \subseteq \theta(\mathcal{J}),[T(\jmath), \theta(\jmath)] \subseteq T(\mathcal{J})$, Albert has observed that, for $\lambda \in \Phi, \lambda^{1 / 2} \notin \Phi, \mathscr{L}(\mathcal{g})_{\lambda}=\lambda^{1 / 2} T(\mathcal{g})+\theta(\mathcal{g})$ with the natural multiplication is again an algebra of type $E_{6}$ over $\Phi$. Finally, adapting a construction of Tits [9], we obtain an algebra $\mathfrak{L}(\mathfrak{E}, \mathfrak{U})$, (5) a Cayley algebra over $\Phi, \mathfrak{A}$ a central simple associative algebra of degree three over $\Phi$ with generic trace form $T(x)$, by defining on the vector space $\theta(\mathfrak{E})+\mathfrak{E} \otimes \mathfrak{A}_{0}, \mathfrak{A}_{0}$ the kernel of $T(x)$, a multiplication $\langle x, y\rangle$ such that

$$
\begin{aligned}
\left\langle D_{1}, D_{2}\right\rangle= & {\left[D_{1}, D_{2}\right] } \\
\left\langle a_{1} \otimes x_{1}, D_{1}\right\rangle= & a_{1} D_{1} \otimes x_{1} \\
\left\langle a_{1} \otimes x_{1}, a_{2} \otimes x_{2}\right\rangle= & {\left[a_{1}, a_{2}\right] \otimes\left(x_{1} \cdot x_{2}-\left(x_{1}, x_{2}\right) 1\right) } \\
& +a_{1} \cdot a_{2} \otimes\left[x_{1}, x_{2}\right]+1 / 3\left(x_{1}, x_{2}\right) D_{a_{1}, a_{3}}
\end{aligned}
$$

for $a_{i} \in \mathfrak{C}, x_{i} \in \mathfrak{A}, D_{a_{1}, a_{2}}=\left[a_{1}, a_{2}\right]_{r}-\left[a_{1}, a_{2}\right]_{l}-3\left[\left(a_{1}\right)_{l},\left(a_{2}\right)_{r}\right]$ in $\mathfrak{C}, a_{r}$ and $a_{l}$ denoting right and left multiplication in $\mathbb{E},[u, v]=u v-v u$, $u \cdot v=\frac{1}{2}(u v+v u),\left(x_{1}, x_{2}\right)=T\left(x_{1} x_{2}\right), 1$ the identity of $\mathfrak{A}, D_{i} \in \theta(\mathfrak{F})$ the derivation algebra of $\subseteq$.

Identifying these algebras with suitable $\Phi$-subalgebras of $\mathscr{L}\left(V, V_{0}\right)$ we have 
TheOREM 4. (a) $\mathcal{L}(\mathcal{g})$ is a Lie algebra of type $E_{6 \mathrm{I}}$ with $\Gamma(\mathfrak{L}(\mathcal{g}))=1$.

(b) $\mathscr{L}(\mathcal{g})_{\lambda}$ is a Lie algebra of type $E_{6 \mathrm{II}}$ with $\Gamma\left(\mathscr{L}(\mathcal{g})_{\lambda}\right)=1$.

(c) $\mathfrak{L}(\mathfrak{S}, \mathfrak{N})$ is a Lie algebra of type $E_{6 \mathrm{I}}$ with $\mathfrak{L}^{*} \cong \mathfrak{B}+\mathfrak{B}^{\prime}, \mathfrak{B}$ equivalent to $\mathfrak{A}$ in the Brauer group.

Theorems 2,3 , and 4 imply that, if $\mathfrak{A}$ is a division algebra over $\Phi$, $\mathfrak{d}, \mathfrak{d}^{\prime}$ exceptional central simple Jordan algebras over $\Phi$, then

CoRollaRy. $\mathscr{L}(\mathcal{J}), \mathfrak{L}\left(\mathcal{J}^{\prime}\right)_{\lambda}, \mathfrak{L}(\mathfrak{S}, \mathfrak{U})$ are nonisomorphic Lie algebras of type $E_{6}$.

We note that the converse of Theorem 4, (a) is true in general, but that we have been unable to establish converses for Theorem 4, (b) and (c) in general.

Special fields. We make the additional assumption in this section that there are no exceptional Jordan division algebras over $\Phi$ and obtain a converse to Theorem 4, yielding

THEOREM $4^{\prime}$. Let $\&$ be a Lie algebra of type $E_{6}$ over $\Phi$. Then

(a) $\mathfrak{L} \cong \mathscr{L}(\mathcal{g})$ if and only if $\Gamma(\mathfrak{L})=1, \mathfrak{L}$ of type $E_{6 \mathrm{I}}$.

(b) $\mathfrak{L} \cong \mathscr{L}(\mathfrak{S}, \mathfrak{U})$ if and only if $\mathfrak{L}^{*}=\mathfrak{B}+\mathfrak{B}^{\prime}, \mathfrak{B}$ of index $\leqq 3$.

(c) If every algebra of type $E_{6 \mathrm{II}}$ over $\Phi$ is split by its canonical $E_{6 \mathrm{I}}$ extension, then $\mathfrak{L} \cong \mathscr{L}(\mathcal{J})_{\lambda}$ if and only if $\Gamma(\mathfrak{L})=1$, $\mathfrak{L}$ of type $E_{6 \mathrm{Ir}}$.

Since for $\Phi$ finite, real closed, or $p$-adic, the condition of Theorem $4^{\prime}$, (c), as well as the general condition of this section, is satisfied, every algebra of type $E_{6}$ over such a field is isomorphic to some $\mathfrak{L}(\mathcal{J}), \mathfrak{L}(\mathcal{J})_{\lambda}$ or $\mathfrak{L}(\mathfrak{S}, \mathfrak{N})$ and, in fact, the latter case can occur only in case $\Phi$ is $p$-adic. One can easily enumerate the nonisomorphic algebras of these kinds, obtaining results agreeing with those in [5], [2], and [7]. If $\Phi$ is an algebraic number field, the condition of Theorem $4^{\prime},(\mathrm{c})$, does not hold and we can show only that the algebras of type $E_{6 \mathrm{I}}$ over $\Phi$ must be isomorphic to some $\mathfrak{L}(\mathcal{J})$ or $\mathfrak{L}(\mathfrak{E}, \mathfrak{A})$.

\section{REFERENCES}

1. R. T. Barnes, On derivation algebras and Lie algebras of prime characteristic, Doctoral Dissertation, Yale University, New Haven, Conn., 1963.

2. E. Cartan, Les groupes reels simples et continus, Ann. Ecole Norm. 31 (1914), 263-355.

3. C. Chevalley and R. D. Schafer, The exceptional simple Lie algebras $F_{4}$ and $E_{6}$, Proc. Nat. Acad. Sci. U.S.A. 36 (1950), 137-141.

4. H. Freudenthal, Beziehungen der $E_{7}$ und $E_{8}$ zur Oktavenebene. I, Indag. Math. 16 (1954), 218-230.

5. D. Hertzig, Forms of algebraic groups, Proc. Amer. Math. Soc., 12 (1961), 657-660. 
6. N. Jacobson, Forms of algebras, Some Recent Advances in the Basic Sciences, Vol. 1, Academic Press, New York, 1966.

7. M. Kneser, Galois-Kohomologie halbeinfacher algebraischer Gruppen iiber padischen Körpern. II, Math. Z. 89 (1965), 250-272.

8. G. Seligman, On the split exceptional Lie algebra $E_{7}$, Mimeographed notes, Yale University, New Haven, Conn.,

9. J. Tits, Algebras alternatives, algebres de Jordan et algebres de Lie exceptionelles, (Summary of an Article in preparation).

The Ohio State University 